\title{
Síndrome de Asperger y autismo de alto funcionamiento: comorbilidad con trastornos de ansiedad y del estado de ánimo
}

\author{
I. Paula-Pérez ${ }^{\text {a,b }}$, J. Martos-Pérez ${ }^{\mathrm{c}}$
}

\author{
SÍNDROME DE ASPERGER Y AUTISMO DE ALTO FUNCIONAMIENTO: \\ COMORBILIDAD CON TRASTORNOS DE ANSIEDAD Y DEL ESTADO DE ÁNIMO
}

\begin{abstract}
Resumen. Introducción. La comorbilidad entre el síndrome de Asperger (SA) y los trastornos del estado de ánimo y de ansiedad parece ser altamente significativa. Por ello, se presenta una revisión de la bibliografía científica más actual que aporte evidencias empíricas a tal hipótesis con el objetivo de plantear una prospectiva de investigación. Desarrollo. La valoración y el diagnóstico del funcionamiento psicosocial analizado en personas con SA o autismo de alto funcionamiento (AAF) confirman una proporción significativa de casos con sintomatología depresiva y ansiosa, y en muchos de ellos se eleva a la categoría de trastornos comórbidos. Conclusión. Es necesaria una mejor formulación diagnóstica ya que las perturbaciones afectivoemocionales y conductuales pueden aparecer enmascaradas como sintomatología asociada al SA/AAF. Identificar y reconocer dicha comorbilidad psiquiátrica mejorará el funcionamiento psicosocial de estas personas. [REV NEUROL 2009; 48 (Supl 2): S31-4]
\end{abstract}

Palabras clave. Autismo de alto funcionamiento. Comorbilidad. Síndrome de Asperger. Trastornos de ansiedad. Trastornos del estado de ánimo.

\section{INTRODUCCIÓN}

Los estudios basados en la clínica sugieren que la depresión y la ansiedad son los trastornos psiquiátricos más comunes en personas con trastorno autista [1-3]. Algunos síntomas relacionados con el estado de ánimo ya se observaron en las descripciones más tempranas del trastorno. Kanner [4] ya sugirió que muchas de las características nucleares del trastorno, especialmente la invarianza ambiental y el repertorio de comportamientos repetitivos, obsesivos y estereotipados, conducían a la manifestación de sintomatología depresiva y ansiosa.

La práctica clínica y educativa también nos revela que las perturbaciones afectivo-emocionales y conductuales son bastante comunes en el síndrome de Asperger (SA) y el autismo de alto funcionamiento (AAF). Es lícito preguntarse, por tanto, si tales perturbaciones afectivas y emocionales no configuran, en algunos casos, un trastorno psiquiátrico comórbido a estos trastornos. Pretendemos examinar los correlatos y consecuencias de la coocurrencia entre SA/AAF y los trastornos de ansiedad y del estado de ánimo. Para ello, se presenta una revisión de la bibliografía científica y de las investigaciones al respecto que aportan evidencias empíricas de esta cuestión de manera que pueda plantearse una futura prospectiva de investigación. La ausencia de estudios con rigor metodológico en nuestro país plantea la necesidad de abrir nuevas líneas de investigación al respecto dentro de nuestras instituciones y servicios de atención a las personas con SA/AAF.

\footnotetext{
Aceptado: 09.01.09.

${ }^{a}$ Departamento de Métodos de Investigación y Diagnóstico en Educación. Universitat de Barcelona. ${ }^{b}$ Posgrado de Trastornos del Espectro Autista. Institut de Ciències de l'Educació. Universitat de Barcelona. Barcelona. ${ }^{c} D E-$ LETREA (Diagnóstico, Evaluación del Lenguaje y Tratamiento del Espectro Autista). Madrid, España.

Correspondencia: Dra. Isabel Paula Pérez. Universitat de Barcelona. Campus Mundet. Edif. Llevant, 2. ${ }^{\circ}$ piso, despacho 275. Pg. Vall d'Hebron, 171. E-08035 Barcelona. E-mail: isabelpaula@ub.edu

(C) 2009, REVISTA DE NEUROLOGÍA
}

\section{DESARROLLO}

En un reciente estudio de Hutton et al [5] se realiza un seguimiento durante, al menos, 21 años de 135 personas diagnosticadas de trastornos del espectro autista en la infancia y con cociente intelectual (CI) superior a 30. En el estudio se examina la aparición de nuevos trastornos psiquiátricos en la vida adolescente o adulta que no estaban presentes en el momento en que se manifestó y diagnosticó el autismo en la infancia. De los pacientes de muestra se localizaron 39 casos con un posible trastorno psiquiátrico nuevo, a quienes se les realizó una valoración psiquiátrica en detalle a través de la entrevista a los padres. El $16 \%$ padeció un nuevo trastorno psiquiátrico confirmado. Un $6 \%$ desarrolló una posible nueva alteración sin diagnóstico firme. Cinco personas se vieron afectadas por un trastorno obsesivocompulsivo y/o catatonía, ocho desarrollaron una alteración afectiva compleja, una persona tuvo trastornos de la alimentación, una presentó un trastorno bipolar que requirió de varios internamientos, y otra persona desarrolló un estado de ansiedad aguda complicada con ideas paranoides, exceso de alcohol y privación de sueño. No se produjo ningún caso de esquizofrenia. Los resultados no muestran una asociación entre los factores pronósticos del desarrollo de autismo y el suceso de nuevas alteraciones psiquiátricas. Las personas con buen funcionamiento (inteligencia no verbal normal) estaban tan afectados como las personas con alteraciones graves que vivían en régimen residencial. Un dato de este estudio que conviene subrayar es que fue común la aparición de nuevos trastornos psiquiátricos, cuando se dieron eventos o cambios vitales negativos (p. ej., en los casos de personas más afectadas, un cambio de residencia, cambio de cuidadores o cambios en las rutinas familiares; en los casos de personas menos afectadas, la pérdida de empleo o la muerte de un ser querido).

La depresión es el trastorno psiquiátrico más común en adolescentes y adultos con SA. Ghaziuddin et al [6], basándose en entrevistas semiestructuradas, la hallaron en el $37 \%$ de la muestra (en 13 de 35 sujetos con SA). Además, tras investigar las historias familiares de los niños con autismo comparando la histo- 
ria familiar de 13 niños autistas con depresión (11 niños y 2 niñas, con un CI de media de 86,2 y una edad promedio de 10,4 años) con 10 niños autistas sin una historia previa recurrente de depresión (9 niños y 1 niña, con un CI de media de 67 y una edad promedio de 10,5 años), estos autores informaron que diez de los niños deprimidos (77\%) tenían una historia familiar de depresión en comparación con tres casos (30\%) del grupo de niños sin depresión. Asimismo, sugirieron que, al igual que en la población normal, los niños con autismo que padecen depresión es más probable que tengan un historial familiar de depresión.

Otros autores $[7,8]$ han informado de la presencia de otros trastornos psiquiátricos. En concreto, se han comparado niños que tenían una historia familiar de trastorno afectivo bipolar con otros niños con trastornos del espectro autista (TEA) sin esa variable familiar, y se han observado claras diferencias en los perfiles sintomatológicos. Los niños sin historia familiar de trastorno afectivo bipolar no presentaban marcadas variaciones cíclicas de comportamiento, manifestaban una menor agitación, menos ansiedad y agresividad, y presentaban un funcionamiento más bajo. En cambio, los niños con historia familiar de trastorno afectivo bipolar mostraban afectos extremos, ciclotimia, intereses obsesivos intensos, trastornos neurovegetativos y regresiones después de un período normal de desarrollo. En particular, existe un gran riesgo de trastorno bipolar en miembros de la familia de personas con SA, e incluso se ha diagnosticado un tercio de familiares de personas con TEA que padecían trastorno afectivo bipolar [9].

Los factores que llevan a las personas con SA a tener un riesgo mayor de síntomas psiquiátricos comórbidos, tales como la ansiedad y la depresión, todavía no se han comprendido del todo. En su estudio de la depresión en personas con autismo, Ghaziuddin et al [2] examinaron los factores que influían en la presencia de depresión, tales como el nivel de inteligencia, la edad, el sexo, los trastornos médicos asociados, el rol de los factores genéticos y los eventos vitales. Estos autores hallaron que las personas con autismo deprimidas presentaban un amplio espectro de síntomas que van desde la irritabilidad a la tristeza, la agresión y el comportamiento suicida. Mientras que la depresión puede diagnosticarse formalmente en personas con un elevado funcionamiento con el mismo criterio que se utiliza para la población general, el diagnóstico puede ser extremadamente complicado en aquellas personas con autismo que tienen graves alteraciones cognitivas y de la comunicación. Los factores que pueden contribuir a la aparición de la depresión son variados. Mientras que en algunos casos puede darse por causalidad, en otros puede resultar de una combinación de factores genéticos o ambientales, o ambos. Incluso, la depresión parece concentrarse en algunas familias con autismo, aunque no existen evidencias de que forme parte de un fenotipo más amplio del autismo. La presencia de otros trastornos médicos puede contribuir a dicha asociación.

Por su parte, Hedley et al [10] investigaron la relación entre los procesos de comparación social y los síntomas depresivos en 36 participantes con SA (34 niños y 2 niñas) con edades comprendidas entre 10 y 16 años. Utilizando una escala de comparación social y un inventario de depresión en niños, observaron una correlación significativa entre síntomas depresivos y la escala de comparación social, lo cual parece sugerir que es un factor a tener en cuenta en la medida en que se proporcionan evidencias de la existencia de una relación entre factores psicosociales y síntomas depresivos. Estos autores consideran que exis- te una necesidad real de desarrollar, investigar y valorar tanto las herramientas de intervención como las de diagnóstico para las personas con SA. Los investigadores sugieren que puede ser efectiva aquella intervención que ayude a las personas con SA a entender, aceptar y apreciar sus diferencias.

El estudio de Meyer et al [11] investigó la posibilidad de que las dificultades emocionales y conductuales en niños de entre 8 y 14 años con SA estén asociadas a los procesos de información y atribución social. Participaron 31 niños con SA y 33 niños con desarrollo normal. Los niños respondieron a viñetas sociales hipotéticas para valorar la información social y los procesos de atribución. También completaron autoinformes sobre las dificultades sociales y el funcionamiento psicológico. Los padres, además, proveyeron de información sobre la competencia social y la presentación clínica. En los resultados del estudio, los niños con SA presentaron una pobre adaptación psicosocial que estaba relacionada con su información social que proporcionaron y los patrones de procesamiento atribucional. Las habilidades cognitivas y cognitivo-sociales se asociaron con la tendencia del procesamiento de la información, pero no se relacionaron con las dificultades emocionales y conductuales. Así, los resultados parecen sugerir que los síntomas comórbidos pueden estar asociados a la percepción, la comprensión y la experiencia social de las personas con SA, reforzando el riesgo significativo de alteraciones psiquiátricas comórbidas y el SA. Al igual que ocurrió en otros estudios previos [6,12], los padres de niños con SA informaron de altas tasas de síntomas más externos (p. ej., la agresividad) y más internos (p. ej., la depresión) en sus hijos. Los propios niños informaron de altas tasas de ansiedad y estrés social, así como una menor satisfacción y competencia en las relaciones interpersonales. Se hallaron, además, indicadores que apuntaban a que los niños con SA eran capaces de informar de sus propias dificultades emocionales y sociales. En primer lugar, los autoinformes de los niños sobre su ansiedad social estaban sistemáticamente correlacionados con los informes de los padres sobre alteraciones en la competencia social de sus hijos. En segundo lugar, los niños informaron de la propia toma de conciencia de sus dificultades sociales, relacionadas directamente con su manera de procesar la información social. En tercer lugar, los autoinformes de los niños sobre sus dificultades emocionales se relacionaron sistemáticamente con su manera de procesar la información social. Cada una de estas observaciones sugería que los niños con SA en este estudio tenían una introspección (insight) suficiente como para autoinformar de sus problemas emocionales y sociales, lo que coincide con los hallazgos de otros investigadores, quienes sugieren que las personas con SA/AAF son capaces de informar sobre sus propias emociones, son conscientes de sus dificultades sociales y tienden a las autoevaluaciones negativas y a la soledad [13].

El estudio de Meyer et al mostraba incluso que los niños con SA eran capaces de interpretar y hacer atribuciones sobre sus situaciones sociales aun cuando manifestaban los típicos patrones de déficit en la teoría de la mente y habilidades sociocognitivas, por lo que puede ser importante comprender las habilidades sociocognitivas y los procesos de atribución social como dominios de actividad potencialmente separables y, en consecuencia, las fortalezas y las debilidades en las medidas cognitivo-sociales pueden no revelar necesariamente cómo los niños con SA entienden y se enfrentan a las situaciones sociales. Algunos niños exhibieron una tendencia negativa en respuesta a viñetas sociales ambiguas. Esta tendencia fue relevante para su 
funcionamiento emocional y conductual. Sobre todo los niños con SA mostraron menos competencia en comprender escenarios sociales y generar respuestas adaptativas en este tipo de tareas comparados con los niños del grupo control. Este estilo de pensamiento puede estar relacionado con fuertes emociones o incapacidad para generar una variedad de respuestas y considerarlas desde todas las perspectivas posibles. La incapacidad para generar respuestas competentes, habilidades pobres en la toma de decisiones y una reactividad emocional fuerte pueden estar interrelacionadas con los déficit que se dan en los niños con $\mathrm{SA}$ en riesgo de padecer un ajuste psicológico insuficiente. Podría concluirse, por tanto, que las personas con autismo pueden variar en su toma de conciencia social y de los otros. En los casos de SA/AAF, una mayor conciencia social y una mayor motivación pueden llevar a una percepción más clara de las propias dificultades en esos dominios sociales. Esta percepción, acompañada de experiencias negativas repetidas e interacciones sociales no exitosas, puede contribuir al desarrollo de aspectos comórbidos psicopatológicos que con el tiempo minan la calidad de vida de estas personas [14].

Cuando se ha analizado el funcionamiento social y psiquiátrico en adolescentes con SA en comparación con adolescentes con trastornos de la conducta [15], el grupo de adolescentes con SA ha mostrado dificultades más intensas en el funcionamiento psicosocial a pesar de las buenas habilidades cognitivas y la falta de retraso en el desarrollo de lenguaje temprano, con altos niveles de trastornos de ansiedad. En ambos grupos se halló: depresión, ideación suicida, temperamento difícil y comportamientos desafiantes. Los autores sugieren que la depresión y la ansiedad en personas con SA refleja una toma de conciencia parcial entre los que tienen trastornos menos graves, y el comportamiento antisocial y peligroso está relacionado con déficit primarios en la empatía. También se argumenta que las alteraciones sociales y la falta de relaciones cercanas hace a las personas con SA más vulnerables a síntomas afectivos secundarios similares a los que pueden aparecer en otras personas con dificultades en la socialización (p. ej., personas con trastornos de la conducta). La historia educativa de los participantes en el estudio (20 adolescentes con SA, 20 adolescentes con trastornos de la conducta) enfatiza la gravedad de las dificultades de adaptación en ambos grupos, junto con una necesidad de recursos educativos especiales en un $80 \%$ en los trastornos de la conducta y un $65 \%$ en el SA. Entre las dificultades sociales más importantes de los individuos con SA destacaba la falta de habilidad para la vida independiente, aunque su inteligencia y funcionamiento en otras áreas eran buenos. Ninguno fue capaz de mantener un trabajo a tiempo parcial. El área en la que ambos grupos mostraron profundas dificultades fue el área de las relaciones sociales, pero con una gran diferencia: esta capacidad era uniformemente baja en todos los sujetos con SA, pero no estaba tan afectada en los sujetos con trastorno de la conducta. Ambos grupos manifestaron una toma de conciencia insuficiente sobre sus dificultades, aunque en un tercio de los sujetos con SA la toma de conciencia fue nula. Asimismo, los resultados del estudio hallaron que los síntomas externos eran comunes en ambos grupos $\mathrm{y}$, en cambio, los internos (como obsesiones y ansiedad) eran más frecuentes en sujetos con SA.

Conviene señalar, tal y como lo hacen Stewart et al [16], que una de las grandes limitaciones metodológicas que tienen los estudios de depresión en personas con autismo radica en delimitar cuáles son los indicadores y criterios que deben utilizarse para diagnosticar trastornos del estado de ánimo dado que el solapamiento existente entre los síntomas de autismo/SA y los de la depresión son importantes. Además, las propias características del autismo y del SA, como son las alteraciones de la comunicación verbal y no verbal, pueden afectar a la manera en cómo la persona expresa dicha sintomatología depresiva. De su trabajo se confirma que los síntomas asociados con el autismo y el SA, tales como las obsesiones y las conductas autolesivas, pueden verse incrementados durante un episodio de depresión; los autores manifiestan la necesidad de desarrollar herramientas específicas para el diagnóstico y para la valoración de la depresión en el autismo y el SA.

En relación con la ansiedad, se ha informado de la prevalencia y la correlación [12] que existen con trastornos del estado de ánimo en niños con SA/AAF de entre 9 y 14 años. Se trabajó con un grupo de 40 niños diagnosticados de autismo cuando tenían entre 4 y 6 años y 19 niños con diagnóstico de SA entre esas mismas edades. En ese momento se les pasó una batería de medidas cognitivas y conductuales, y seis años después se contactó con sus familias (cuando los niños tenían una edad media de 12 años) y se evaluaron posibles evidencias de trastornos de ansiedad y del estado de ánimo. En comparación con la muestra de 1.751 niños de la comunidad, los niños con SA/AAF mostraron una tasa mayor de ansiedad y problemas depresivos. Dichos problemas tuvieron un impacto significativo en su adaptación global, sin que existieran, no obstante, diferencias en el número de problemas de ansiedad y del estado de ánimo entre los niños con SA/AAF. En el estudio de Gillott et al [17], los investigadores compararon niños autistas de alto funcionamiento con dos grupos control en medidas de ansiedad y preocupación social. El estudio explora la naturaleza de la ansiedad en niños con autismo de alto funcionamiento, y para ello se administraron medidas estandarizadas de ansiedad general y de preocupación social por separado a grupos de 15 niños con AAF, 15 niños con un desarrollo normal y 15 niños con alteraciones específicas del lenguaje, de entre 8 y 12 años. Los resultados confirmaron que los niños con autismo manifestaban una mayor ansiedad en ambas medidas (ansiedad y preocupación social). Los trastornos de ansiedad más prevalentes fueron el trastorno de ansiedad generalizada, la ansiedad por separación y la fobia específica.

\section{CONCLUSIONES}

La depresión es un trastorno frecuente en las personas con autismo y depende de varios factores. Además de los síntomas generales de tristeza y pérdida de interés en las actividades, las personas con autismo pueden manifestar la depresión o la ansiedad de otras formas, de una manera atípica. Los indicadores conductuales más utilizados para el diagnóstico de la depresión han sido: humor deprimido indicado por el propio sujeto o a través de la observación, disminución del interés o el placer en todas o casi todas las actividades, pérdida o aumento de peso significativo o cambios significativos del apetito, insomnio/hipersomnia, agitación, fatiga o pérdida de energía, sentimientos de inutilidad o de culpa excesivos e inapropiados, disminución de la habilidad para pensar/concentrarse, indecisión, pensamientos recurrentes de muerte, ideación suicida e intentos o planes de suicidio.

Las dificultades en habilidades de la teoría de la mente (atribuir estados mentales y emocionales en los demás y en ellos mismos) no proporcionan inmunidad contra la depresión. Las personas con autismo y con lenguaje pueden comunicar mejor sus sín- 
tomas depresivos que los que tienen un funcionamiento más bajo, y parece ser que las personas con SA/AAF son más vulnerables a la depresión que las personas con autismo y retraso mental asociado. Aunque ello se relaciona con la toma de conciencia de sus propias limitaciones, aún tenemos pocas evidencias empíricas al respecto. Una forma de inicio de un proceso depresivo en las personas con autismo puede ser la falta de interés por sus temas o actividades objeto de obsesiones, rituales, etc.

Los estudios e investigaciones realizadas están presentando distintas limitaciones metodológicas: no hay estudios que diferencien la ansiedad y los trastornos del estado de ánimo con los síntomas de TEA. El trastorno autista a menudo implica la manifestación de características tales como fobias específicas, hiperactividad, obsesiones, estereotipias, etc., que hace complicado diferenciarlas de síntomas afectivos o ansiosos, sobre todo en aquellas personas con alteraciones importantes en la comunicación; no disponemos de instrumentos de valoración válidos para la evaluación de los trastornos de ansiedad y los trastornos del estado de ánimo en personas con autismo de bajo funcionamiento $\mathrm{y}$ con pocas habilidades verbales; no existen investigaciones suficientemente validadas sobre los correlatos y los factores de riesgo de ansiedad y los trastornos del estado de ánimo y los TEA; no disponemos de datos empíricos con relación a qué sín- tomas tempranos del autismo pueden correlacionarse con problemas de ansiedad y depresivos; no se han llevado a cabo estudios con grandes poblaciones de personas con SA y los estudios que existen son muy variables por lo que respecta al tamaño de la muestra, el período en que se estudia, las edades, los criterios diagnósticos, etc., lo cual no permite generalizar los datos.

La revisión documental realizada sugiere que debería examinarse y sistematizarse el impacto de la depresión en los resultados a largo plazo de las personas con autismo al objeto de determinar cuál es la presentación de los síntomas, especialmente en aquellos pacientes que sufren de marcados déficit en la comunicación. También nos permite considerar que sería razonable y efectivo intervenir sobre las atribuciones, el autoconcepto y el procesamiento de la información social para el desarrollo de tratamientos en niños y adolescentes con SA/AAF.

Es importante identificar y reconocer la comorbilidad psiquiátrica, pues ello mejoraría sustancialmente el funcionamiento psicosocial de estas personas. Para ello puede requerirse de una mejor formulación diagnóstica, ya que suele producirse un retraso en el diagnóstico de trastornos afectivos comórbidos debido a que las perturbaciones afectivo-emocionales y conductuales aparecen enmascaradas como sintomatología asociada al SA/AAF.

\section{BIBLIOGRAFÍA}

1. Howlin P. Autism: preparing for adulthood. London: Routledge; 1997.

2. Ghaziuddin M, Ghaziuddin N, Greden J. Depression in persons with autism: implications for research and clinical care. J Autism Dev Disord 2002; 32: 299-306.

3. Gillott A, Frurniss F, Walter A. Anxiety in high-functioning children with autism. Autism 2001; 5: 277-86.

4. Kanner L. Autistic disturbances of affective contact. Nervous Child 1943; 2: $217-50$.

5. Hutton J, Goode S, Murphy M, Le Couteur A, Rutter M. New-onset psychiatric disorders in individual with autism. Autism 2008; 12: 373-90.

6. Ghaziuddin M, Greden J. Depression in children with autism/pervasive developmental disorders: a case-control family history study. J Autism Dev Disord 1998; 32: 299-306.

7. DeLong R. Children with autistic spectrum disorder and a family history of affective disorder. Dev Med Child Neurol 1994; 36: 674-87.

8. DeLong R, Dwyer JT. Correlation of family history with specific autistic subgroups: Asperger's syndrome and bipolar affective disease. J Autism Dev Disord 1988; 18: 593-600.

9. Piven J, Chase GA, Landa R, Wzorek M, Gayle J, Cloud D, et al. Psychiatric disorders in the parents of autistic individuals. J Am Acad Child Adolesc Psychiatr 1991; 30: 471-8.
10. Hedley D, Young R. Social comparison processes and depressive symptoms in children and adolescents with Asperger syndrome. Autism 2006; 10: 139-53.

11. Meyer JA, Mundy PC, Van Hecken AV, Durocher JS. Social attribution processes and comorbid psychiatric symptoms in children with Asperger syndrome. Autism 2006; 10: 383-402.

12. Kim JA, Szatmari P, Bryson S E, Streiner DL, Wilson F. The prevalence of anxiety and mood problems among children with autism and Asperger syndrome. Autism 2000; 4: 117-32.

13. Barnhill GP. Attributional style and depression in adolescent with Asperger syndrome. Journal of Positive Behaviours Interventions 2001; 3: $175-82$

14. Tantam D. Psychological disorder in adolescent and adults with Asperger syndrome. Autism 2000; 4: 47-62.

15. Green J, Gilchrist A, Burton D, Cox A. Social and psychiatric functioning in adolescents with Asperger syndrome compared with conduct disorder. J Autism Dev Disord 2000; 30: 279-93.

16. Stewart ME, Barnard L, Pearson J, Hasan R, O'Brien G. Presentation of depression in autism and Asperger syndrome: a review. Autism 2006; 101: 103-16.

17. Gillott A, Furniss F, Walter A. Anxiety in high-functioning children with autism. Autism 2001; 5: 277-86.

\section{ASPERGER'S SYNDROME AND HIGH-FUNCTIONING AUTISM: \\ COMORBIDITY WITH ANXIETY AND MOOD DISORDERS}

Summary. Introduction. The comorbidity between Asperger's syndrome and affective and anxiety disorders seems to be meaningful. Therefore, it is presented a revision of the most recent scientific literature to provide empiric data with the aim to set out a research prospective. Development. Assessment and diagnosis of the psychosocial performance confirm a meaningful proportion of cases with affective and anxious symptomatology, being converted into disorders some of them. Conclusion. It is necessary a better diagnosis because the affective and behavioural disturbances can be hidden as Asperger's syndrome and high-functioning autism associated symptomatology. Identify and recognize this comorbidity will improve the psychosocial capacity of these persons. [REV NEUROL 2009; 48 (Supl 2): S31-4]

Key words. Anxiety disorders. Asperger's syndrome. Comorbidity. High-functioning autism. Mood disorders. 